\title{
EFFICACY OF OPEN CAP SPLINT VERSUS SPLIT ACRYLIC SPLINT IN MANAGEMENT OF PEDIATRIC MANDIBULAR FRACTURES
}

\author{
Abdel Aziz Baiomy Abdullah Baiomy*, Mostafa Ibrahim Fayad** and Ihab M Atito***
}

\begin{abstract}
Objective: This prospective randomized clinical study was designed to evaluate the efficacy of open cap splint versus split acrylic splint in management of pediatric mandibular fractures. Patients and Methods: Twelve pediatric patients with symphysis, parasymphysis, and body of mandibular fractures treated by closed reduction and fixation were randomly selected. They were divided randomly into two equal groups: group (I) patients were treated by open cap splint and group (II) patients were treated by the split acrylic splint. Postoperative clinical and radiographic evaluations were performed to assess complications, occlusal stability, pain on a visual analogue scale (VAS), maximum mouth opening, and the overall results of fixation up to 6 months. Results: Fracture healing was optimal in all cases, except two cases in group I presented malunion of the fractured bone. Satisfactory dental occlusion was observed in all cases, except two cases in group I, where they had mild to moderate occlusal derangement. Other parameters including maximal mouth opening, and pain level were comparable. Postoperative radiographic evaluation showed a complete union of the fractured segments at the end of six months in all cases. Conclusion: The results of the current study showed that, the open cap splint and split cap splint provided enough stability for proper bone healing, establishment of optimal occlusion, and early return to normal function with superiority for split cap splint.
\end{abstract}

\section{INTRODUCTION}

Amongst the facial fractures, the fracture of the nasal bone is the most common type, while the mandibular fractures are the following most common type of fractures reported in hospitalized pediatric patients ${ }^{(1-3)}$. Major causes of pediatric mandibular fractures are fall from a height, road traffic accidents, child hyperactivity ${ }^{(3-5)}$.
Management of fracture injuries to the facial skeleton in children is still a challenging clinically due to un co-operation of the child and a growing facial bones so it presents great difficulty in choosing appropriate treatment ${ }^{(1)}$

The treatment modalities of pediatric fracture range from conservative noninvasive through closed reduction and immobilization methods, to open re-

\footnotetext{
* Ass. Professor of Oral and Maxillofacial Surgery, Faculty of Dental Medicine (Assiut) - Al-Azhar University ** Ass. Professor of Removable Prosthodontics, Faculty of Dental Medicine (Cairo- Boys)- Al-Azhar University. *** Lecturer of Removable Prosthodontics, Faculty of Dental Medicine (Cairo- Boys)- Al-Azhar University
} 
duction with internal fixation depending upon the type of fracture, the skeletal stage, and dentition development ${ }^{(6)}$. Closed reduction is preferred in children because of the bone is elastic, and mandibular cortex is thin and less dense in comparison to the adults, and the presence of tooth buds. Also, disruption of the periosteal envelop of the mandible during open reduction may have an unpredictable effect on the growth of the mandible ${ }^{(6,7)}$.

Most of the pediatric fractures are greenstick type, so conservative approach is preferred as the fracture heals fast and the child grows normally. Another advantage of closed reduction is that it can be performed on an outpatient basis under local anesthesia, so more patient compliance and cooperation is attained with fewer complications ${ }^{(5-7)}$. Majority of body and parasymphysis fracture in children are minimally displaced because of elasticity of bone. Slight occlusal discrepancies resulting from lack of perfect reduction may be corrected with the eruption of permanent teeth. Non displaced body or symphysis fracture without malocclusion can be treated by closed reduction, soft diet, and avoidance of physical activity. Exact method used for immobilization depends upon child's age and stage of dental development ${ }^{(4,8)}$.

In pediatric patient bone plating may results in injury to the developing tooth buds. Another problem with internal fixation is second surgery for removal of the hardware ${ }^{(9,10)}$

Currently, open reduction and fixation with resorbable osteosynthesis plates and screws may be used in children, but these systems are not devoid of complications as poor mechanical property and lack of ease in handling of these type of resorbable implants $^{(6,11)}$. Therefore, Non-surgical management (observation, exercises, maxillomandibular fixation, elastics) are popular because of minimal complications and good outcomes ${ }^{(9)}$.

The occlusal acrylic splint is one of the conservative modalities used in the closed reduction of the pediatric fracture. It has several advantages such as its ease of fabrication, patient compliance, preservation of periodontal tissue integrity, and ease in assessing the dental occlusion after reduction (7-

9). There are two types of occlusal acrylic splints, original open cap splint and split acrylic splint ${ }^{(8)}$. However, there are little studies compared between them in efficacy aspect as fixation modalities in pediatric fractures. Accordingly, the present study was conducted to compare between the two types of acrylic splint for conservative treatment of mandibular fractures in children.

\section{PATIENTS AND METHODS}

\section{I- Patients Selection:}

This study was carried out on twelve pediatric patients (seven males and five females). Patients ages were ranged between eight years old and twelve years old (average ten years). The study extended from February 2015 to February 2019. The patients were randomly selected from those patients attending at the out-patients clinic of oral and maxillofacial surgery, Faculty of Dental Medicine, Al-Azhar University (Assuit). Informed consents were obtained from the parents of all patients before carrying out any study procedure.

Patient with mixed dentition and having symphysis, parasymphysis fracture, or body of mandible fractures were included in the study. On the other hand, mandibular angle fracture, condylar and other mandibular fractures associated with panfacial fractures were excluded. The diagnosis was attained after clinical examination and radiographic findings.

\section{Preoperative Clinical and Radiological Ex- amination}

The patient had a chief complaint of pain and restriction in mouth opening. The patients were well oriented and conscious. No history of loss of consciousness, no bleeding ear or nose were reported. Extraoral examination revealed that there 
was a slight swelling related to the body of the mandible. There was limited mouth opening owing to pain and muscle spasm. Clinically a displaced fracture was evident. Intraoral examination revealed the state of occlusion and tooth structures. Some cases had a sublingual hematoma and lacerated lip which treated by primary suturing and preoperative medication. Orthopantomograph (OPG) confirmed fracture lines and the relation of primary teeth and teeth bud to fracture line (Fig.1 A,B).

\section{Splint fabrication:}

Occlusal acrylic splints were fabricated in Removable Prosthodontics laboratories, Faculty of Dental Medicine, Al-Azhar University. Under local anesthesia, upper and lower arch impressions were made, then two sets of casts were subsequently poured. The casts were split with a saw at the fracture site and the segments brought into normal alignment and stabilized. An acrylic open cap splint was fabricated on this modified cast (fig. 2A). The split acrylic splint designed as acrylic open cap splint but with two wires gagged $4 \mathrm{~mm}$ in both distal ends and swing lock shape in midline after splitting at midline (fig.2B).

\section{Patients Grouping:}

Patients were divided into two equally divided groups according to the type of acrylic splint:
- Group I: Included six pediatric patients treated by open cap splint with circum-mandibular wiring.

- Group II: Included six pediatric patients treated by split acrylic splint with circum- mandibular wiring.

\section{Surgical procedures:}

Under general anaesthesia displaced fractures were reduced and acrylic splints in both groups were placed over mandible after reduction. Then , circummandibular wiring performed by an mandibular awl was used to pass the 26 gage wire percutaneously from the submandibular region and exited in the lingual side close to the alveolus and clamped intraorally, then the tip of the awl was railroaded till the lower border of the mandible was felt, and then the awl passed on the buccal side in close proximity to the alveolus (fig..3 A \& B). The awl and the excess wire within were removed after cutting the desired length of wire intra-orally and then the wires twisted to tighten the splint in position. Care was taken to avoid injury to the mental nerve and the facial artery. In split acrylic splint wire at midline was twisted to tighten two parts of buccal plate of splint. Excessive pulling was avoided as the child mandibular cortex was relatively thin (fig.3 C \& D).

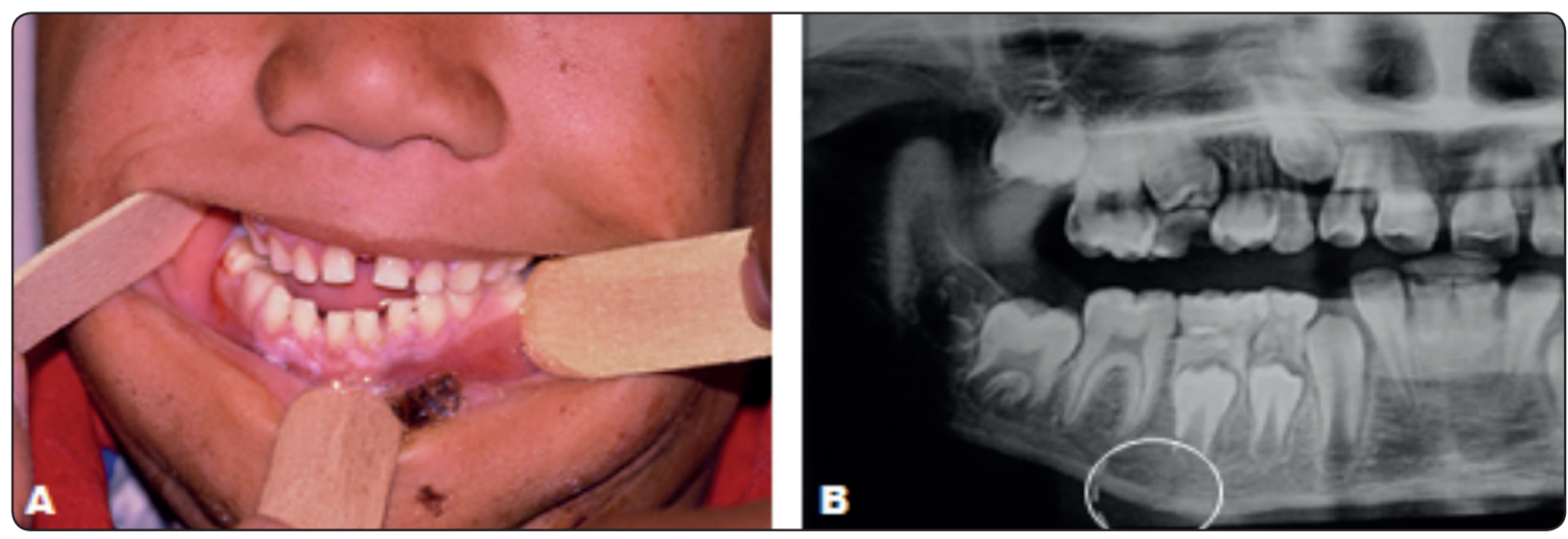

Fig. (1) A) Preoperative Intraoral clinical photograph showing limited mouth opening and occlusal derangement. B) Preoperative OPG showing body fracture . 


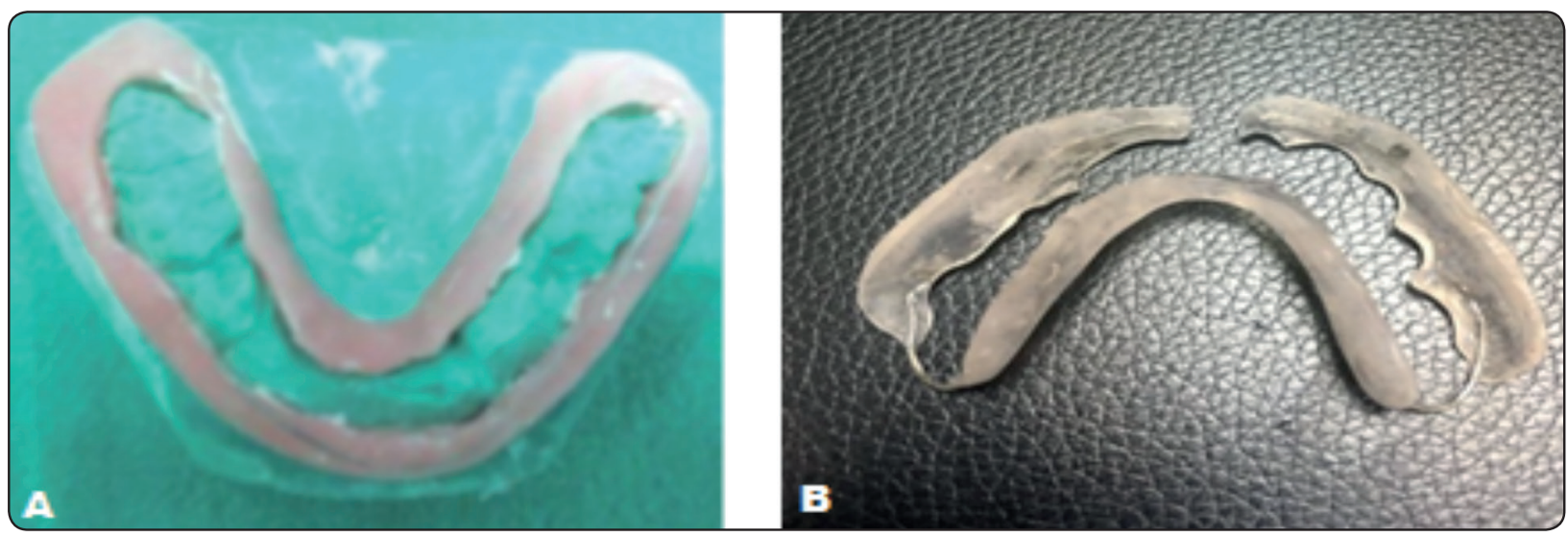

Fig. (2) A) Open cap splint (B) Split acrylic cap splint
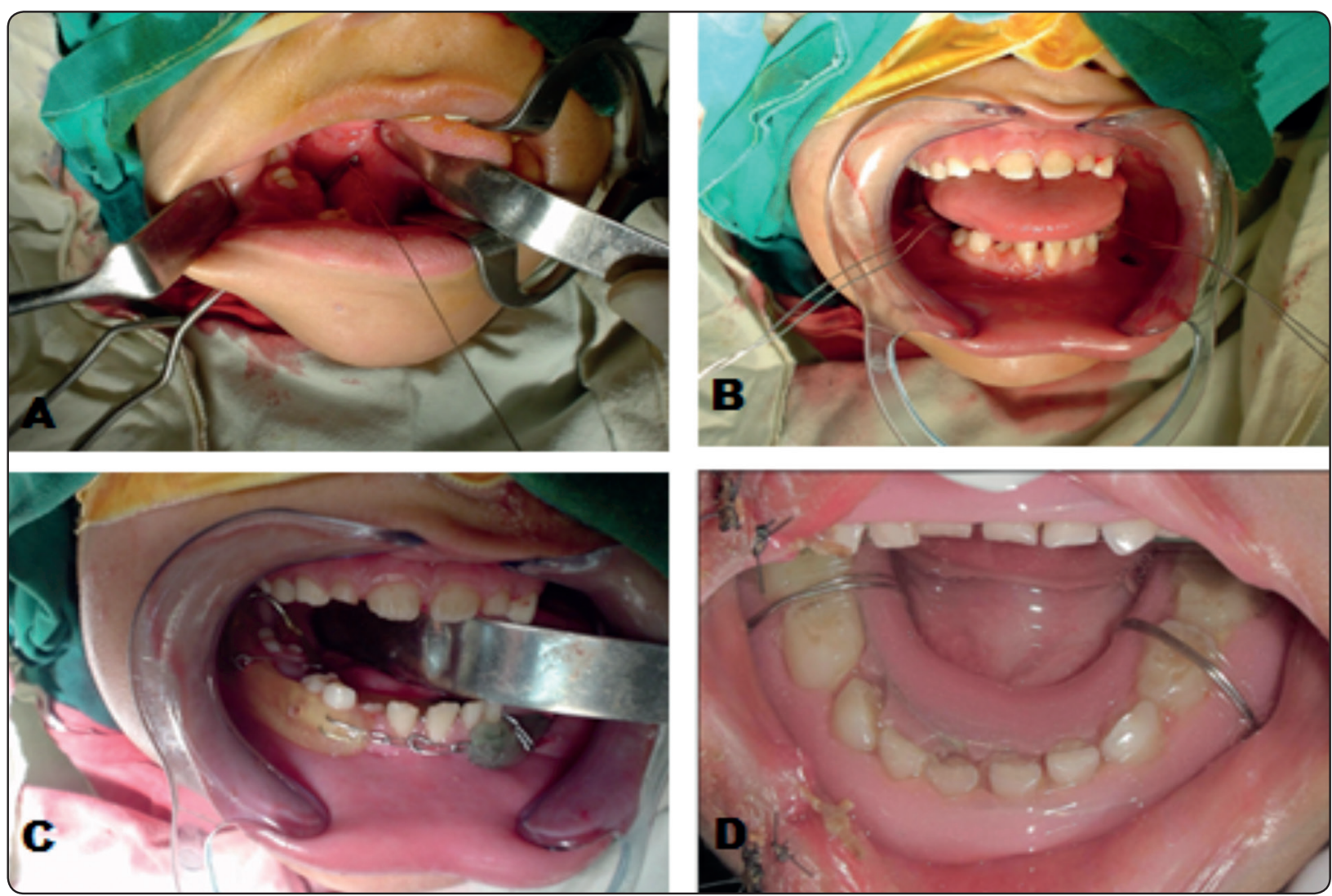

Fig. (3) (A) An awl exited in the lingual side (B) The wires passed buccally and lingually $\quad$ (C) Split cap splint in situ after reduction (D) Open cap splint in situ after reduction 
Parents were advised for the maintenance of oral hygiene of the child and kept his child on a soft/ liquid diet. Systemic antibiotics and anti-oedematous medications were prescribed. The patients were schaduled to followed up clinically after 24 hours, third postoperative day, seventh day and then weekly for four weeks and then monthly intervals for six months. On the 4th postoperative week, the circummandibular wiring and acrylic splint was removed. The patients were followed up radiographically with OPGs, at the immediate postoperative period, after one month, and at six months.

Postoperatively, patients were evaluated for the range of mouth opening, level of pain on a visual analogue scale (VAS), with a value from 0 (no pain) to 10 (strongest pain or discomfort)), occlusal derangements, malunion, nonunion and infection \& the observation were recorded. Descriptive statistics and t-test was done for comparison between mean values of VAS and mean values of mouth opening measurements in both groups. The level of significance for the statistical tests was set at $\mathrm{p}<0.05$. All statistical measures were performed through the statistical package for social studies (SPSS) version 19 for windows.

\section{RESULTS}

In the present study, age of the patients ranged from 8 to 12 years with a mean of 10 years. Of the twelve selected patients, four patients had fracture sustained parasymphysis, five patients had fracture body of the mandible and three patients had fracture symphysis of the mandible. All children showed satisfactory fracture healing with evidence of union of fracture segments at the end of six months clinically and radiographically without any complications except two cases in group I showed malunion at fracture line with occlusion derangement.

\section{Occlusion}

In group I, preoperative dental occlusion was disturbed in $95 \%$ of cases $(n=5)$. After splint removal, two patients $(n=33.3 \%)$ were found to had an occlusal discrepancy that was resolved by minor occlusal grinding. While, in group II, preoperative dental occlusion was disturbed in $100 \%$ of cases (n $=6$ ). After splint removal, there was no evidence of any malocclusion in all cases. Therefore, there was a statistically significant difference between the two study groups in terms of occlusal discrepancy (fig.4A).

\section{Pain (VAS):}

There was no statistically significant difference between the two study groups regarding to the VAS scores to assess pain, except for the VAS scores at the first week follow-up. The VAS scores at firstweek follow-up were lower in group II $(3.10 \pm 1.45)$ than in group I $(4.30 \pm 2.72)$; the difference was statistically significant (table 1 ).

\section{Mouth opening measurement:}

With regard to the mouth opening, there was no statistically significant difference between the two groups through two weeks of follow-up. At the fourth week follow-up, mouth opening was higher in group II $(38.45 \pm 7.6)$ as compared to group I $(33.25 \pm 4.7)$; this difference was statistically significant (table 2).

\section{Radiographic assessment:}

On the radiographic assessment of the postoperative radiographs, adequate anatomic reduction was found in group II with no disturbance of the superior border and good lower border alignment. In group I, there were two patients in whom mal-union with mal-alignment was visualized on the radiographs at the lower border. This is because of the instability of bone fragments during the fixation period (fig.4B). 
TABLE (1) Compression between VAS scores means of both groups at different intervals

\begin{tabular}{|l|c|c|c|}
\hline Variable & $\begin{array}{c}\text { Group I } \\
\text { mean } \pm \text { SD }\end{array}$ & $\begin{array}{c}\text { Group B } \\
\text { mean } \pm \text { SD }\end{array}$ & P-value \\
\hline Preoperative & $8.15 \pm 1.50$ & $7.85 \pm 1.27$ & 0.498 \\
\hline Postoperative & $6.45 \pm 2.19$ & $5.35 \pm 1.81$ & 0.092 \\
\hline 1-week follow-up & $4.30 \pm 2.72$ & $3.10 \pm 1.45$ & $0.022 *$ \\
\hline 4-week follow-up & $0.45 \pm 1.32$ & $0.50 \pm 1.43$ & NS \\
\hline 6 month follow-up & 0 & 0 & \\
\hline
\end{tabular}

SD, standard deviation; NS, not significant

* statistically significance when $p<0.05$

TABLE (2) Comparison between means of mouth opening measurements of both groups at different intervals

\begin{tabular}{|c|c|c|c|}
\hline Variable & $\begin{array}{c}\text { Group I } \\
\text { mean } \pm \text { SD }\end{array}$ & $\begin{array}{c}\text { Group B } \\
\text { mean } \pm \text { SD }\end{array}$ & 0.547 \\
\hline Preoperative & $15.04 \pm 6.4$ & $15.72 \pm 7.2$ & 0.056 \\
\hline Postoperative & $19.36 \pm 8.1$ & $21.95 \pm 5.3$ & 0.435 \\
\hline 1-week follow-up & $27.61 \pm 6.5$ & $29.73 \pm 6.9$ & $0.013 *$ \\
\hline 4-week follow-up & $33.25 \pm 4.7$ & $38.45 \pm 7.6$ & 0.447 \\
\hline 6 month follow-up & $37.32 \pm 7.5$ & $38.81 \pm 6.3$ & \\
\hline
\end{tabular}

SD, standard deviation;

* Statistically significance when $p<0.05$

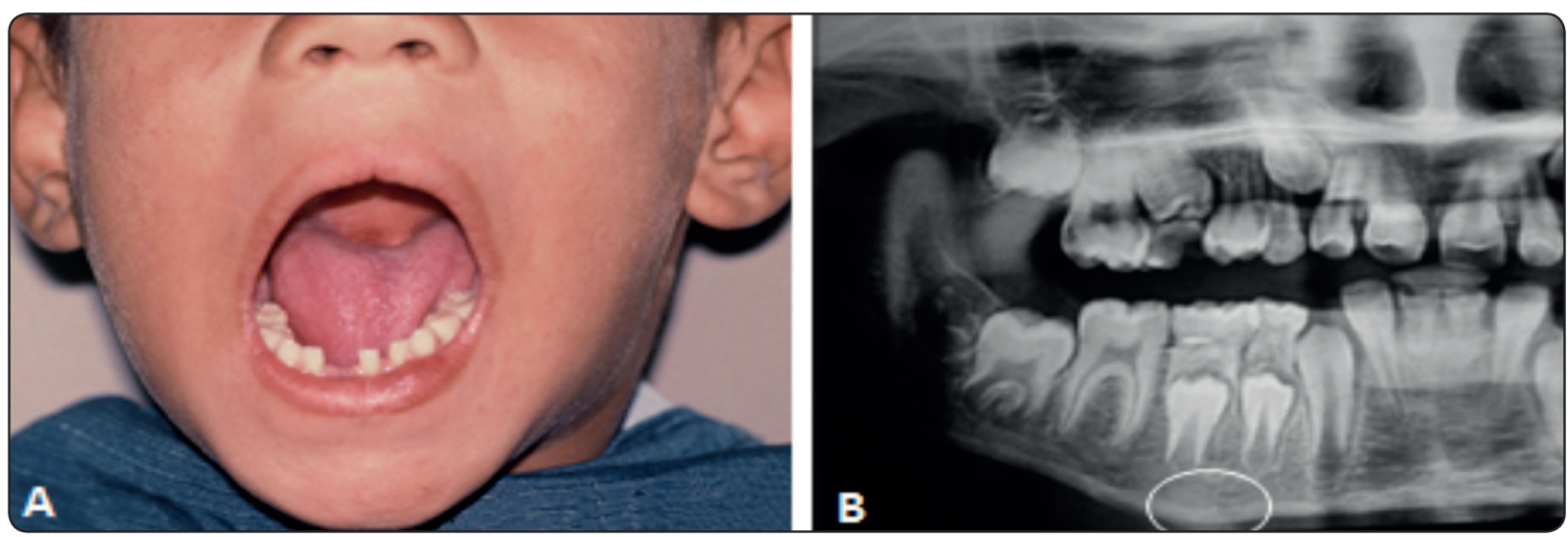

Fig. (4) A) Postoperative intraoral clinical photograph showing maximum mouth opening B) postoperative OPG at six months showing complete bone healing at the fracture site in group II. 


\section{DISCUSSION}

The current study was conducted to evaluate the efficacy of open cap splint versus split acrylic splint as fixation modality in the pediatric mandibular fracture. It showed that healing of fracture site was satisfactory in all the 12 patients with the evidence clinically and radiographically of union of the fracture segments at the end of the study. There was uneventual postoperative complications except 2 cases in group I showed malunion at fracture line with occlusal derangement.

The present study was carried out on twelve pediatric patients with ages were ranged between 8 year old and 12years old (average 10 years). Hence, the conservative treatment modality used in this study was the only alternative in pediatric patients in which mixed dentition is found. This is in agreement with Singh et al ${ }^{(1)}$ and Hegab ${ }^{(12)}$ studies.

The present study showed that both of open cap splint and split acrylic splint with circummandibular wiring resulted in satisfactory occlusion and stability, they were easy to apply and removal, reduced the operating time, and caused minimal trauma. These results are in agremment with the results of Hegab ${ }^{(12)}$, Bhola et al. ${ }^{(13)}$, and Kale et al. ${ }^{(14)}$ studies. They used non-surgical treatment in their studies, comprising a compression acrylic splint associated with circum-mandibular fixation. However, no one of these studies compared between the two types of splints as in the present study.

Little complications were observed among the treated patients. This is in agreement with Aizenbud et al. ${ }^{(15)}$ who mentioned that complications per se are very rare in pediatric trauma due to the child's greater osteogenic potential, faster healing rate. Yuvaraj V.et al ${ }^{(16)}$ encountered tear in floor of mouth resulting in a hematoma in floor of mouth in 3 out of $22(13.6 \%)$ pediatric patients, but in the present study, none of the patients developed a postoperative hematoma.
In the present study, postoperative occlusal evaluation showed a statistically significant difference at the end of study between the two study groups in terms of occlusal discrepancy. While VAS scores presented an early decrease in pain level in group II than group I. Hence, there is a statistical significant difference between both groups at the first week postoperatively. In the same side, Mouth opening measurements showed early improvement in group II than group I at 4 weeks follow up. Difference between both groups in these parameters may explained by the instability of bone fragments during the fixation period in group I.

Also, postoperative radiographs presented adequate anatomic reduction was found in group II with no disturbance of the superior border and good lower border alignment. In group I, there were two patients in whom mal-union with malalignment was visualized on the radiographs at the lower border. This is because of the instability of bone fragments during the fixation period. This explanation is in agrement with Torgersen et al ${ }^{(17)}$ conclusions statement. They stated that mal-union could be caused by improper reduction, inadequate occlusal alignment, and inadequate stability of the fracture. Therefore, split acrylic splint may be better than open cap splint in mandibular fracture reduction and fixation aspects. Where, the occlusion was satisfactory, with early relieve of pain and early MO improvement with no mal-union occurrence.

\section{CONCLUSION}

In conclusion, results of the present study proved that both open cap splint and split acrylic splint with circum-mandibular wiring are satisfactory methods for mandibular fracture fixation in the pediatric patient. Although the functional outcomes pain (VAS score) at first-week follow-up and interincisal mouth opening at fourth week follow-up were found to be better in split acrylic splint patient group. Moreover, the radiographic assessment was also better in split acrylic splint group than open cap splint group as the inferior border was better aligned with no superior border distraction. 


\section{CONFLICTS OF INTEREST}

There are no conflicts of interest.

\section{REFERENCE}

1. Singh AK, Sharma NK, Verma V, Pandey A. Open Cap Splint Fixation with Circum Mandibular Wiring still best method in Management of Pediatric Mandibular Fracture. Journal of dentofacial sciences. 2014; 3(4): 55-8.

2. Dodson TB. Mandibular fracture in children, OMS Knowledge Update, Vol. 1, part II, pp.1995; 95-107.

3. Slida, Matsuya T. Pediatric maxillofacial fractures; their aetiological characters and fracture patterns. Journal of Cranio Maxillofacial Surgery 2002; 30:4; 237-41.

4. Posnick JC, .Wells M, Pron GE, Alpert B. Pediatric facial fractures: evolving patterns of treatment. Journal of Oral and Maxillofacial Surgery 1993; 51:8; 836-45.

5. Zimmermann CE, Troulis MJ, Kaban LB. Pediatric facial fractures: Recent advances in prevention, diagnosis and management. Int J OralMaxillofac Surg 2006; 35:2-13.

6. Eppley BL. Use of resobable plates and screws in pediatric facial fractures. J Oral Maxillofac Surg; 2005,63:385-91.

7. Amit A, Deepshikha A, Deepti D, Vinay D. Management of Pediatric Mandibular Fracture using Acrylic cap splint \& Circum-mandibular Wiring - A Report of 12 cases. Int J Orthop Traumatol Surg Sci 2015:1(1):16-9.

8. Holland AJ, Broome C, Steinberg A, Cass DT. Facial fractures in children .Pediatr Emerg Care 2001; 17:157-60.

9. Valiati R, Ibrahim D, Abreu ME, Heitz C, de Oliveira RB, Pagnoncelli RM. The treatment of condylar fractures: To open or not to open? Acritical review of this controversy. Int J Med Sci. 2008; 5(6):313-8.
10. Shetty V, Atchison K, Leathers R, Black E, Zigler C, Belin TR. Do the benefits of rigid internal fixation of mandible fractures justify the added costs? Results from a randomized controlled trial. J Oral Maxillofac Surg. Nov 2008;66 (11):2203-12.

11. Senel FC, Tekin US, Imamoglu M, Trabzon, Kirikkale. Treatment of mandibular fractures with bioresorbable plates in an infant: report of a case. Oral Surg Oral Med Oral Path Oral Radiol Endod; 2006;101:448-50.

12. Hegab A. Management of mandibular fractures in children with a split acrylic splint: a case series. Br J Oral Maxillofac Surg 2012;50:e93-5

13. Bhola N, Jadhav A, Borle R, Khemka G, Adwani N, Bhattad M. Lateral compression open cap splint with circummandibular wiring for management of pediatric mandibular fractures: a retrospective audit of 10 cases. Oral Maxillofac Surg 2014;18:65-82.

14. Kale TP, Urologin SB, Kapoor A, Lingaraj JB, Kotrashetti SM. Open cap splint with circummandibular wiring for management of pediatric mandibular parasymphysis/ symphysis fracture as a definitive treatment modality: a case series. Dent Traumatol 2013;29:410-5

15. Aizenbud D, Hazan-Molina H, Emodi O, Rachmiel A. The management of mandibular body fractures in young children. Dental Traumatol; 2009; 25:565-70.

16. Yuvaraj V, Thomas S, Dal Singh, Prabhu S, Cyriac S, Thakur G. Awl versus intravenous cannula stillete in circummandibular wiring - a prospective comparative study. Oral Maxillofac Surg; 2011; 15:21-5.

17. Torgersen S, Tornes K. Maxillofacial fractures in a Norwegian district. Int J Oral Maxillofac Surg 1992; 21(6): $335-8$ 